\title{
Resolving the stellar outskirts of six Milky Way-like galaxies beyond the Local Group
}

\author{
A. Monachesi ${ }^{1}$, E. F. Bell ${ }^{2}$, D. J. Radburn-Smith ${ }^{3}$, B. Harmsen ${ }^{2}$, \\ R. S. de Jong ${ }^{4}$, J. Bailin ${ }^{5,6}$, B. W. Holwerda ${ }^{7}$ and D. Streich ${ }^{4}$ \\ ${ }^{1}$ Max Planck Institute for Astrophysics, Karl-Schwarzschild-Str. 1, Garching D-85748, \\ Germany \\ ${ }^{2}$ Department of Astronomy, University of Michigan, 311 West Hall, 1085 South University \\ Ave., Ann Arbor, MI 48109, USA \\ ${ }^{3}$ Department of Astronomy, University of Washington, Seattle, WA 98195,USA \\ ${ }^{4}$ Leibniz-Institut für Astrophysik Potsdam, D-14482 Potsdam, Germany \\ ${ }^{5}$ Department of Physics and Astronomy, University of Alabama, Box 870324, Tuscaloosa, AL, \\ 35487, USA \\ ${ }^{6}$ National Radio Astronomy Observatory, P.O. Box 2, Green Bank, WV, 24944, USA \\ ${ }^{7}$ University of Leiden, Sterrenwacht Leiden, Niels Bohrweg 2, NL-2333 CA Leiden, The \\ Netherlands
}

\begin{abstract}
Models of galaxy formation in a hierarchical universe predict substantial scatter in the halo-to-halo stellar properties, owing to stochasticity in galaxies' merger histories. Currently, only few detailed observations of galaxy's halos are available, mainly for the Milky Way and M31. The Galaxy Halos, Outer disks, Substructure, Thick disks and Star clusters (GHOSTS) HST survey is the largest study to date of the resolved stellar populations in the outskirts of disk galaxies and its observations offer a direct test of model predictions. Here we present the results we obtain for six highly inclined nearby Milky Way-mass spiral galaxies. We find a great diversity in the properties of their stellar halos.
\end{abstract}

Keywords. galaxies: halos, galaxies: spiral, galaxies: evolution, galaxies: photometry

We constructed the stellar halo color/metallicity and density profiles of red giant branch stars out to $\sim 70 \mathrm{kpc}$ along the minor axis of each of the massive galaxies from the GHOSTS survey (Radburn-Smith et al. 2011). Their stellar density profiles can be broadly characterized by a range of power law functions $\Sigma \sim r^{-\alpha}$ where $2<\alpha<4$ (Harmsen et al. in prep.). For most galaxies there is substantial scatter around a single power law with coherent bumps and wiggles in the profiles, indicative of stellar halo substructure. Half of the galaxies present a clear negative color gradient in their halos, reflecting a declining metallicity; the other have no significant color or population gradient (Monachesi et al. 2016a). Our results along the minor axis agree reasonably well with predictions from stellar halo models built entirely from accretion events; we find little evidence that an in situ halo is needed to reproduce observations. However, we showed in Monachesi et al. (2016b) that the in situ halo component is prominent primarily along the major axis for edge-on galaxies, where GHOSTS observations have limited radial coverage. A quantitative comparison between our minor axis profiles and the minor axis profiles from cosmological hydrodynamical simulations is required in order to put stronger constraints on the in situ halo models (Monachesi et al. in prep.). 